\title{
Impactos na qualidade de vida dos pacientes pós transplante hepático
}

Impacts on the quality of life of patients after hepatic transplantation

\author{
Impactos en la calidad de vida de los pacientes tras el trasplante hepático
}

Glenda Stephanie Araújo da Silva ${ }^{1 *}$, Bianca Martins da Costa e Castro², Caio César da Costa Franco $^{3}$, Diego Rodrigues Escher ${ }^{1}$, Lucas da Costa Franco ${ }^{1}$, Lucas Martins dos Santos Tannús², Marcela Mapa Linhares ${ }^{1}$, Maria Eduarda Moreira Martins da Costa ${ }^{4}$, Paulo Henrique Oliveira Van Der Maas Cruz ${ }^{1}$, Wagner Pablo Corrêa ${ }^{5}$.

\section{RESUMO}

Objetivo: Este artigo buscou realizar uma revisão da literatura abordando como o transplante hepático pode influenciar de maneira significativa na qualidade de vida (QV) dos pacientes acometidos por doenças hepáticas. Revisão bibliográfica: Diversos elementos podem afetar a QV de pacientes transplantados, como a idade, o uso de imunossupressores e complicações pós-operatórias, como a síndrome metabólica e repercussões cardiovasculares. Trabalhos apontam que a QV melhora significativamente após o transplante de fígado (TF), em todas as idades, produzindo melhorias no bem-estar físico, proporcionando menor restrição nas atividades sociais e de lazer. Embora os pacientes que recebem TF apresentem melhorias na QV, muitos relatam dificuldades de retornar ao trabalho, lidar com sintomas abdominais, sistêmicos e fadiga, desenvolvimento de hipertensão, diabetes, hiperlipidemia, ansiedade e depressão. Considerações finais: Dessa forma, faz-se necessário o acompanhamento dos pacientes por uma equipe de saúde multidisciplinar para que o transplantado seja assistido durante todo o processo, aumentando assim, a sobrevida, e consequentemente promovendo uma melhoria no bem-estar.

Palavras-chave: Transplante hepático, Qualidade de vida, Tolerância ao transplante.

\begin{abstract}
Objective: This article sought to conduct a literature review addressing how liver transplantation can significantly influence the quality of life (QOL) of patients with liver disease. Bibliographic review: Several elements can affect the QOL of transplant patients, such as age, the use of immunosuppressants and postoperative complications, such as the metabolic syndrome and cardiovascular repercussions. Studies show that QOL improves significantly after liver transplantation (TF), at all ages, producing improvements in physical well-being, providing less restriction on social and leisure activities. Although patients who receive TF experience improvements in QOL, many report difficulties in returning to work, dealing with abdominal, systemic symptoms and fatigue, development of hypertension, diabetes, hyperlipidemia, anxiety and depression. Final consideration: Thus, it is necessary to monitor patients by a multidisciplinary health team so that the transplant is assisted throughout the process, thus increasing survival, and consequently promoting an improvement in well-being.
\end{abstract}

Key words: Liver transplantation, Quality of life, Transplantation tolerance.

\section{RESUMEN}

Objetivo: Este artículo buscaba realizar una revisión de la literatura que abordara cómo el trasplante de hígado puede influir significativamente en la calidad de vida (CV) de los pacientes con enfermedad hepática. Revisión bibliográfica: Varios elementos pueden afectar la CV de los pacientes trasplantados, como la edad, el uso de inmunosupresores y las complicaciones postoperatorias, como el síndrome metabólico y las

\footnotetext{
${ }_{1}^{1}$ Centro Universitário de Caratinga (UNEC), Caratinga - MG. *E-mail: araujo.glendaa@gmail.com

2 Universidade de Itaúna (UIT), Itaúna - MG.

${ }^{3}$ Universidade Federal de Juiz de Fora (UFJF), Governador Valadares - MG.

${ }^{4}$ IMEPAC Centro Universitário (IMEPAC), Araguari - MG.

${ }^{5}$ Faculdade de Medicina do Vale do Aço (UNIVACO), Ipatinga - MG.
} 
repercusiones cardiovasculares. Los estudios muestran que la calidad de vida mejora significativamente después del trasplante de hígado (TF), en todas las edades, produciendo mejoras en el bienestar físico, proporcionando menos restricciones en las actividades sociales y de ocio. Aunque los pacientes que reciben TF experimentan mejoras en la calidad de vida, muchos informan dificultades para regresar al trabajo, lidiar con síntomas abdominales, sistémicos y fatiga, desarrollo de hipertensión, diabetes, hiperlipidemia, ansiedad y depresión. Consideraciones finales: Por tanto, es necesario el seguimiento de los pacientes por parte de un equipo sanitario multidisciplinar para que el trasplante sea asistido durante todo el proceso, aumentando así la supervivencia y, en consecuencia, favoreciendo una mejora del bienestar.

Palabras clave: Trasplante de hígado, Calidad de vida, Tolerancia al trasplante.

\section{INTRODUÇÃO}

As patologias hepáticas avançadas são responsáveis por alterações metabólicas, por perda das funções musculares, respiratória e desnutrição, além de outros sintomas sistêmicos relacionados com a hepatopatia. A associação desses sintomas é responsável por induzir uma deficiência motora de forma global e à inatividade física, interferindo de forma negativa na realização das atividades de vida diária e na QV dos pacientes portadores de doença hepática (DH) em fase avançada (AGUIAR MIF, et al., 2018).

$\mathrm{A} \mathrm{DH}$, quando caracterizada de forma irreversível, está relacionada à alta taxa de morbimortalidade, com diminuição significativa do bem-estar do paciente, refletindo em sintomas físicos e impactos psicossociais, sendo o TF indicado como a única alternativa de manutenção da vida. Em 2013, o Brasil ocupou o segundo lugar no ranking de países com maior número de transplantes hepáticos. Entre 2005 e 2015, foram realizados cerca de 14.800 transplantes de fígado (AGUIAR MIF, et al., 2018).

Como resultado dos avanços tecnológicos e o uso de novos imunossupressores, o tempo de sobrevida dos pacientes submetidos ao TF aumentou. No entanto, algumas complicações devido ao transplante ainda são ameaçadoras à saúde dos pacientes, como a síndrome metabólica, que é uma complicação comum decorrente do transplante hepático (WHANG Z, et al., 2018). Além disso, a DH corresponde ao quarto lugar geral entre as principais causas de morte em pessoas entre 45 a 54 anos (VOLK ML e HAGAN M, 2011)

O TF é um processo amplo e complexo, que demanda uma abordagem de uma equipe multiprofissional para poder assistir o paciente no processo de adaptação e recuperação, melhorando, assim, os resultados e sucesso pós cirúrgico. Isso é crucial para que haja uma melhora nos aspectos funcionais e sociais do transplantado (GANGERI L, et al., 2018). Além do mais, o elevado grau de adesão e comprometimento do paciente ao longo dos tempos, é responsável por aumentar a taxa de sobrevida em cerca de 20 anos de pelo menos $50 \%$ dos pacientes que realizaram o procedimento (KRENZIEN F, et al.,2017).

No Brasil, o procedimento de TF do doador para o receptor, obedece ao critério de severidade do comprometimento hepático, através da utilização do escore Model for End-Stage Liver Disease (MELD), independentemente do tempo em que os pacientes estão na lista de espera. A pontuação do MELD é baseada em uma equação que tem como variáveis os níveis séricos de creatinina, bilirrubina e a razão normalizada internacional (INR) para o tempo de protrombina. A pontuação final pode ter uma variação de 6 a 40 pontos, em que a evolução da doença e a indicação terapêutica, podem ser analisadas juntamente com o prognóstico do paciente no prazo de 3 meses, predição de mortalidade e sobrevivência após o transplante, além de servir como um parâmetro de avaliação da qualidade de vida (AGUIAR MIF, et al., 2016).

As indicações para o TF, atualmente, são preferencialmente relacionadas à hepatite $\mathrm{C}$ e doença hepática alcoólica, correspondendo a $40 \%$ do total de candidatos adultos que esperam pelo procedimento (BARCELOS S, et al., 2008). Dados estes corroboram com o estudo realizado por Aguiar MIF, et al. (2016), em que analisaram 150 receptores e as patologias mais prevalentes foram decorrentes do uso abusivo de álcool, correspondendo a $38,7 \%$ dos pacientes analisados, seguido por hepatite $\mathrm{C}$, que correspondeu a $28 \%$, e a cirrose sendo $14,7 \%$, além de outras doenças como o carcinoma hepatocelular, doença de Wilson e hepatite B. 
O objetivo do presente trabalho consistiu em realizar uma revisão narrativa da literatura abordando como o TF pode influenciar de maneira significativa na qualidade de vida dos pacientes.

\section{REVISÃO BIBLIOGRÁFICA}

\section{Alteração da qualidade de vida}

A Qualidade de Vida $(\mathrm{QV})$ é um fator considerável na realização do TF. Se define como uma construção multifacetada do indivíduo, incluindo sua cultura e sistema de valores em relação aos seus padrões, suas expectativas e seus objetivos (AGUIAR MIF, et al., 2018; WHANG Z, et al., 2018). Diversos elementos podem afetar a QV de pacientes transplantados, como a idade, uso de imunossupressores e complicações pósoperatórias, como, por exemplo, a síndrome metabólica e repercussões cardiovasculares (WHANG Z, et al., 2018).

Existem inúmeros instrumentos e questionários voltados para a análise da QV após TF, aplicado em pacientes com DH crônica de qualquer etiologia. O Short Form-36-Item Health Survey (SF-36) é o questionário mais usado para análise da QV após TH. Investiga aspectos físicos, sociais e psicológicos. É composto de 36 itens, divididos em 8 domínios de saúde física e mental. São eles: funcionamento físico, papel-físico, dor corporal, saúde geral, vitalidade, funcionamento social, papel-emocional e bem-estar psicológico. Possui alta confiabilidade e sensibilidade a mudanças (GEORGIOS P, et al., 2017).

Outro instrumento amplamente utilizado nas pesquisas sobre QV do paciente que recebe TF, é o Liver Disease Quality of Life (LDQOL). É um instrumento traduzido e validado para a população brasileira, que permite avaliação de vários aspectos da QV, além de ser aplicável em pacientes com diferentes graus de gravidade de $\mathrm{DH}$ e que foram submetidos ao transplante (AGUIAR MIF, et al., 2018). São avaliados 12 domínios, sendo memória, interação social, preocupação, isolamento, estigma da $\mathrm{DH}$, função sexual e problemas sexuais, variáveis com melhores resultados pós transplante (AGUIAR MIF, et al., 2016).

Apesar da existência dos formulários para avaliação da QV pós TF, existem limitações em seu uso. O SF36, por exemplo, por se tratar de questões padronizadas, acaba perdendo domínios individualmente e importantes (THIEL C, et al., 2013). Ainda, nota-se uma escassez de estudos para análise da QV dos pacientes submetidos ao TF no Brasil, sendo oportuno a realização de mais pesquisas para entendimento dos impactos e consequências, bem como para traçar estratégias de acompanhamento dos pacientes em questão, oferecendo melhor assistência ao cuidado (AGUIAR MIF, et al., 2016).

Embora existam limitações nos estudos, os trabalhos apontam que a QV melhora significativamente após o TF, em todas as idades, produzindo melhorias no condicionamento físico, proporcionando menor restrição nas atividades sociais e de lazer, mais energia e menos dor e desconforto (ABERG F, 2020). Segundo resultados de uma revisão sistemática realizada por Yang LS, et al. (2014) a QV dos pacientes que se submeteram ao TF é superior ao estado pré-transplante, por pelo menos, 20 anos. Mais de $80 \%$ das pesquisas apontaram para melhorias no funcionamento físico e $60 \%$ mostraram melhorias no funcionamento mental e social do paciente (WHANG Z, et al., 2018).

Os resultados apontam para melhoria da interação social nos pacientes transplantados, além de melhora no autocuidado, no funcionamento sexual, independência e mobilidade, redução do isolamento social, minimização de sentimentos como depreciação, frustração e desencorajamento, queda na irritação e aumento da comunicação e afetividade com família e amigos (AGUIAR MIF, et al., 2016; MATOS MM, 2011; DABROWSKA-BENDER M, et al., 2016).

Cerca de $45 \%$ dos receptores de TF realmente retornam aos trabalhos após o transplante, e observaram que a QV, em relação ao convívio social, aumenta significativamente quando associado ao emprego, sendo este um indicador para maiores pontuações nos questionários de QV. Além disso, também foi analisado que a falta de emprego pós TF parece ser fortemente influenciada por fatores socioeconômicos anteriores do que pela gravidade da doença, tanto pré transplante, quanto pós transplante (BOWNIK H e SAAB S, 2009). 
Embora os pacientes que recebem TF apresentem melhorias na QV, muitos relatam resultados não tão satisfatórios, principalmente quando investigados até 6 meses após o transplante, quando descrevem limitações sexuais, que de acordo com Sorrel JH, et al. (2006) a disfunção sexual permanece por muito tempo no período após o transplante. Além disso, os pacientes relatam dificuldades de retornar ao trabalho, lidar com sintomas abdominais, sistêmicos e fadiga, desenvolvimento de hipertensão, diabetes, hiperlipidemia, ansiedade e depressão. É importante salientar que os padrões de sono saudáveis não são recuperados com frequência, o que interfere diretamente na capacidade de realizar as atividades habituais (AGUIAR MIF, et al., 2016; FLOR MJN, 2015; ABERG F, 2020; BURRA P e FERRARESE A, 2019; CASANOVAS T, et al., 2016; GANGERI L, et al., 2018; TELLES-CORREIA D, et al., 2009).

\section{Impactos na saúde mental}

Pacientes que apresentam ansiedade e depressão antes do TF são mais propensos a desenvolverem e terem maiores escores dessas comorbidades psiquiátricas após o transplante. A identificação e o tratamento ativo dessas patologias podem trazer benefícios para o paciente transplantado melhorando a QV (LINS L, et al., 2017). Dessa maneira, é importante uma avaliação detalhada da saúde mental por uma equipe multiprofissional, afinal, o TF é a melhor possibilidade terapêutica para pacientes com DH avançada, fazendo, portanto, necessária a implantação do suporte psiquiátrico para garantir ao paciente um bom estado de saúde mental (GUERREIRO-COSTA LNF, et al., 2019).

Um estudo realizado por Guerreiro-Costa LNF, et al. (2016) feito com 248 pacientes que estavam na lista de espera para TF em um Hospital privado em Salvador e em um Hospital Universitário, observou-se que cerca de $40 \%$ dos inscritos na lista de espera apresentavam comorbidades psiquiátricas de diferentes etiologias, e que poderiam afetar de forma negativa o resultado do procedimento e consequentemente uma diminuição no bem-estar daquele paciente.

Segundo Aberg F (2020), cerca de um terço dos receptores de TF desenvolvem transtornos de humor e ansiedade no primeiro ano após o procedimento e até $40 \%$ dos transplantados possuem características de depressão, que está fortemente associada a uma diminuição na qualidade de vida. Além disso, o transtorno do estresse pós-traumático foi associado a uma sobrevida prejudicada.

Flor MJN (2015), analisou a QV de 256 pacientes adultos submetidos ao TF, sendo que 15 (5,9\%) apresentaram ansiedade e $4(1,6 \%)$ desenvolveram depressão severa após o procedimento, e observaram que a idade superior a 45 anos no momento do transplante, depressão, ansiedade e enxerto de doador falecido foram fatores que influenciaram a QV relacionada à saúde mental no pós-operatório do TF.É importante considerar que pacientes transplantados podem sofrer com a ansiedade devido a preocupações com os efeitos colaterais do tratamento com imunossupressor, dificuldade de se adaptar ao processo e condições pós TF, além da angústia excessiva com o risco de morte devido a rejeição do órgão (AGUIAR MIF, et al., 2018).

Os pacientes portadores de hepatite $C$ apresentam um risco de seis vezes maior de suicídio, assim como o surgimento de sintomas de ansiedade e depressão que podem resultar numa diminuição da QV quando comparados com a população em geral (GUERREIRO-COSTA LNF, et al., 2019).

\section{Limitações dos pacientes pós TF}

Casanovas T, et al. (2016) fizeram um estudo com 156 pacientes em que a taxa de sobrevivência pós TF foi de $84,7 \%$, foi possível observar que o padrão de sono saudável nunca se recupera. A má qualidade do sono é conhecida por influenciar as condições físicas e cognitivas do paciente. A falta de sono está relacionada com a incapacidade de o transplantado participar das atividades de vida diária com êxito. Além disso, este estudo mostra que algumas limitações físicas nunca serão totalmente recuperadas mesmo após o TF.

A fadiga é outra limitação que afeta cerca de até $60 \%$ dos receptores de TF, e sua gravidade está relacionada intimamente com a qualidade do sono, depressão e ansiedade. É um sintoma mais físico do que mental, e está relacionada com complicações pós TF ou com regime de imunossupressão. Neste mesmo 
estudo foi possível observar que a fadiga presente nos pacientes antes do procedimento melhorou cerca de $20 \%$ pós TF (ABERG F, 2020).

A disfunção sexual sofre variação individual, e está presente em muitos pacientes mesmo após o TF (ABERG F, 2020). Ho JK, et al. (2006), em seus estudos relataram que $26 \%$ das mulheres e $23 \%$ dos homens que receberam TF apresentaram problemas com a libido, $26 \%$ das mulheres e $33 \%$ dos homens tiveram dificuldade de atingir o orgasmo. Dessa forma, a porcentagem da disfunção sexual aumentou nos receptores hepáticos sendo um fator limitante na QV dos pacientes.

\section{Observações a longo prazo}

Na revisão sistemática realizada por Yang LS, et al. (2014), evidenciaram que o TF foi associado a uma melhora global entre os estudos analisados sobre QV geral pós-transplante, e que esta permaneceu superior ao estado pré-transplante quando comparada por um período de pelo menos 20 anos.

No estudo de Burra $P$ e Ferrarese $A$ (2019), sobre o acompanhamento dos pacientes transplantados à longo prazo foram observadas flutuações positivas na QV. A melhoria nesse aspecto foi explicada devido a capacidade que o paciente tem de alcançar uma nova estabilidade psicoemocional, recuperar suas atividades de vida diária, incluindo o retorno ao trabalho.

Rodrigues RTS, et al. (2008), concluíram que os pacientes transplantados, continuam na dependência dos serviços de saúde, implicando diretamente na sua rotina de vida. Estes dados corroboram com achados que referem que a melhora imediata observada na QV não é mantida ao longo do tempo.

Whang Z, et al. (2018), observaram que a síndrome metabólica (SM) é uma complicação comum após o TF e tem uma incidência significativamente maior em pacientes que realizaram o procedimento quando comparada à população geral. Entre os participantes desse estudo, 46,94\% apresentaram SM após o TF, sendo que o subgrupo dos pacientes obesos apresentaram maior incidência $(53,74 \%)$ e dislipidemia exibiu a menor incidência. Apesar de ser uma complicação que pode levar a lesões vasculares, neste estudo foi concluído que a SM não teve impacto específico na QV dos pacientes a longo prazo.

\section{Estigma social}

O estigma social da DH reflete diretamente em diferentes áreas da vida do paciente. Porém, com o sucesso do procedimento e recuperação da cirurgia, os estigmas físicos da doença, como o ressecamento de pele, aumento do volume abdominal devido à ascite, edema e a ginecomastia nos homens desaparecem e a pessoa pode voltar a ter uma vida mais próxima do normal. Um estudo observou uma redução da ocorrência no estigma da $\mathrm{DH}$ entre os pacientes avaliados antes do procedimento e após o procedimento, com maior destaque para vergonha da aparência, correspondendo a uma redução de $96,7 \%$ para $59,3 \%$. Dessa forma, houve uma melhora significativa dos níveis de QV nesse domínio após um ano do transplante (AGUIAR MIF, et al., 2018).

Nos estudos de Burra P e Ferrarese A (2019), o estigma social permaneceu baixo mesmo após o TF, visto que a $\mathrm{DH}$ interfere fortemente nas relações sociais e na autoimagem do paciente transplantado, acarretando comportamentos contrários relacionados à saúde. Quando o TF é incapaz de resolver este problema, o paciente deve ser incentivado a retornar ao trabalho e ao convívio social. Ainda nesse mesmo estudo, observaram que o emprego após o TF é um marcador do estado funcional após a cirurgia e tem sido bastante associado a um ganho elevado na qualidade de vida.

\section{CONSIDERAÇÕES FINAIS}

Após a revisão da literatura sobre este tema, pode-se concluir que os pacientes submetidos ao TF apresentam alterações em vários aspectos relacionados à qualidade de vida. Depressão, ansiedade, disfunção sexual, preocupação excessiva, fadiga, síndrome metabólica, limitações físicas e sociais que estão intimamente relacionados com os indivíduos antes, durante e após o TF. As manifestações relacionadas à qualidade de vida dependem da existência de doenças pré-existentes, da severidade da $\mathrm{DH}$ e de fatores 
psicossociais. Dessa forma, faz-se necessário o acompanhamento dos pacientes por uma equipe multiprofissional para que o transplantado seja assistido durante todo o processo, aumentando assim, a sobrevida, e consequentemente promovendo uma melhoria no bem-estar.

\section{REFERÊNCIAS}

1. ABERG F. Quality of life after liver transplantation. Best Practice \& Research Clinical Gastroenterology, 2020; 46-47.

2. AGUIAR MIF, et al. Aspectos psicossociais da qualidade de vida de receptores de transplante hepático. Texto e Contexto Enfermagem, 2018; 27(2): 1-11.

3. AGUIAR MIF, et al. Gravidade da doença hepática e qualidade de vida no transplante de fígado. Acta Paul Enfermagem, 2016; 29(1): 107-114.

4. BARCELOS S, et al. Transplante Hepático: repercussões na capacidade pulmonar, condição funcional e qualidade de vida. Arq Gastroenterol, 2008; 45(3): 186-191.

5. BOWNIK H, SAAB S. Health-Related Quality of Life After Liver Transplantation for Adult Recipients. Liver Transplantation, 2009; 15(11): 42-49.

6. BURRA P, FERRARESE A. Health-Related quality of life in liver transplantation: another step forward. Transplant International, 2019; 32: 792-793.

7. CASANOVAS T, et al. Identifying Improved and Non-improved Aspects of Health-related Quality of Life After Liver Transplantation Based on the Assessment of the Specific Questionnaire Liver Disease Quality of Life. Transplantation Proceedings, 2016; 48: 132-137.

8. DABROWSKA-BENDER M, et al. Assessment of the Quality of Life in Patients After Liver Transplantation as an Important Part of Treatment Results. Transplantation Proceedings, 2016; 48(5): 1697-1702.

9. FLOR MJN. Avaliação da qualidade de vida do paciente transplantado hepático. Dissertação (Mestrado em Ciências Médico-Cirúrgicas). Universidade Federal do Ceará, Fortaleza, 2015.

10. GANGERI L, et al. A Longitudinal Investigation of Posttraumatic Growth and Quality of Life in Liver Transplant Recipients. Progress in Transplantation, 2018; 1-8.

11. GEORGIOS P, et al. Posttraumatic Stress Disorder, Quality of Life, and the Subjective Experience in Liver Transplant Recipients. Progress in Transplantation, 2017;1-7.

12. GUERREIRO-COSTA LNF, et al. Mental disorders and quality of life in patients awaiting liver transplantation. Arquivos de Gastroenterologia, 2019; 56(4): 339-343.

13. HO JK, et al. Sexual health after orthotopic liver transplantation. Liver Transplant 2006;12: 1478 e84.

14. KRENZIEN F, et al. The elderly liver transplant recipients: anxiety, depression, fatigue and life satisfaction. $Z$ Gastroenterol, 2017; 55(6): 557-563.

15. LINS L, et al. Oral Health and Quality of Life in Candidates for Liver Transplantation. Transplantation Proceedings, 2017; 49: 836-840.

16. MATOS MM. Impacto da doença e do transplante hepático na qualidade de vida de doentes com cirrose: estudo exploratório. Tese de Mestrado - Cadernos de Saúde. Universidade Católica Editora, Lisboa, 2011.

17. RODRIGUES RTS, et al. Estudo preliminar sobre a qualidade de vida e sintomatologia depressiva de pacientes em fase pré e pós-transplante hepático. Arquivos Brasileiros de Ciências da Saúde, 2008; 33(2): 74-79.

18. SORREL JH, BROWN JR. Sexual functioning in patients with end-stage liver disease before and after transplantation. Liver Transplantation, 2006; 12(10): 1472-1477.

19. TELLES-CORREIA D, et al. When Does Quality of Life Improve After Liver Transplantation? A Longitudinal Prospective Study. Transplantation Proceedings, 2009; 41: 904-905.

20. THIEL C, et al. Contributors to individual quality of life after liver transplantation. European Journal of Clinical Investigation, 2013; 43(1): 11-19.

21. VOLK ML, HAGAN M. Organ Quality and Quality of Life After Liver Transplantation. Liver Transplantation, 2011; 17: 1443-1447.

22. WHANG Z, et al. Impact of metabolic syndrome on quality of life of liver transplant recipients. Journal of International Medical Research, 2018; 0(0): 1-10.

23. YANG LS, et al.. Transplante de fígado: uma revisão sistemática da qualidade de vida em longo prazo. Liver Int; 2014; 34: 1298. 